(c) SHABALINA A. V.

UDC 542.87

DOI: $10.20333 / 2500136-2021-2-107$

\title{
Electrochemical aptasensors for diagnostics and analysis of biological samples. Important aspects
}

A. V. Shabalina

Tomsk State University, Tomsk 634050, Russian Federation

Abstract The transition to personalized medicine requires a continuous improvement in the quality of diagnostics, and, consequently, improvement and efficiency of the use of diagnostic tools. Biosensors have high potential in the field of clinical diagnosis, especially in the early stages of diseases. The use of postgenomic technologies, in particular the use of DNA-aptamers, which are recognizing biomolecules, as part of biosensors, in combination with highly sensitive methods of analysis, such as electrochemical ones, can further increase the potential of using biosensor systems. Because it becomes possible to determine the presence of target objects (for example, tumor markers or markers of other diseases) with high sensitivity and selectivity of analysis. However, despite the rapid growth in the number of scientific research and publications in the field of the development of electrochemical biosensors based on aptamers (aptasensors), at the moment the commercialization of the products obtained occurs slowly and to a low level.

The market of biosensors is still dominated by enzymes and antibodies-based sensors that are standardized in the industry. This is probably due to the fact that the business originated from enzymes/antibodies utilization at first. But also the general lack of knowledge on the performance of aptasensors in research and in practical use plays an important role [1]. The latter, inter alia, is a consequence of the lack of a proven theoretical base to date, of confirmed key mechanisms and patterns governing the processes of obtaining the response of an electrochemical aptasensor. However, it is obvious that the confirmed knowledge of the key patterns and mechanisms of the biosensor response formation in the presence of the target object will allow a sufficiently high degree of control of this process to optimize the analysis procedure and increase its efficiency and stability of the aptasensor making it suitable for the practical use.

A large variety of approaches are used for new electrochemical aptasensors development. Among them there are different signal amplification strategies, nanoparticles and other biomolecules addition to the aptasensor's construction, more complicated signal formation schemes, and so on [2]. So, the first important aspect is the fact that every new-developed approach requires theoretical consideration and deep study of the sensing mechanism.

Before developing an aptasensor for the commercial purposes, one should consider the target task and the specific requirements, namely sampling and samples' preparation, analyte's type and the targeted concentration range, the possible composition of the matrix, noise, the final cost of the device, etc. [2-5]. Thus, two next important aspects are the necessity to take into account the real samples' specificity and the request for the minimal possible price.

The last important point that needs to be highlighted is the analytical signal of the electrochemical aptasensor itself. Normally, for the entire electrochemical response recorded in the form of dependences of one or more parameters on the programmed effect (current, potential, frequency), one feature is selected (called an analytical signal), by which all the data is analyzed. More frequently, to detect the presence of the target analyte, the magnitude of the selected feature is compared for the response of the biosensor before and after contact with the test sample (for example, [6]). However, the responses are complex and are composed of many features. The signal can be either a nontrivial feature, or it might be their pair or a triple combination. Identification of the informative part of the response is the work of obtaining/generating an aptasensor signal.

So, in the present work, four important aspects that need to be considered during electrochemical aptasensor development were pointed out. Not all of them are widely presented in the literature, but still, they require the attention of the researchers.

Key words: electrochemical aptasensor, aptamer, oncological diseases, voltammetry, impedance measurement.

Conflict of interest. The authors declare the absence of obvious and potential conflicts of interest associated with the publication of this article.

Citation: Shabalina AV. Electrochemical aptasensors for diagnostics and analysis of biological samples. Important aspects. Siberian Medical Review. 2021; (2):107. DOI: 10.20333/2500136-2021-2-107

\section{Литература / References}

1. Moreno M. Aptasensors. In: Encyclopedia of Analytical Science, 3rd ed. Elsevier Inc.; 2019:5109. DOI: 10.1016/B978-0-12-409547-2.13934-4

2. Rozenblum GT, Pollitzer IG, Radrizzani M. Challenges in Electrochemical Aptasensors and Current Sensing Architectures Using Flat Gold Surfaces. Chemosensors. 2019;7(4):57-70. DOI: 10.3390/chemosensors7040057

3. Abnous K, Mohammad Danesh N, Alinezhad Nameghi M, Ramezani M, Alibolandi M, Lavaee P, Mohammad Taghdisi S. An Ultrasensitive Electrochemical Sensing Method for Detection of Microcystin-LR Based on Infinity-Shaped DNA Structure Using Double Aptamer and Terminal Deoxynucleotidyl Transferase. Biosensors and Bioelectronics. 2019;(144):111674-111680. DOI: 10.1016/j.bios.2019.111674

4. Fatemi F. Design and Fabrication of a Label-Free Aptasensor for Rapid and Sensitive Detection of Endoglucanase. International Journal of Biological
Macromolecules. 2020;(148):276-283. DOI: 10.1016/j.ijbiomac.2020.01.030

5. Li Y, Liu D, Zhu C, Shen X, Liu Y, You T. Sensitivity Programmable Ratiometric Electrochemical Aptasensor Based on Signal Engineering for the Detection of Aflatoxin B1 in Peanut. Journal of Hazardous Materials. 2020;(387): 122001-122009. DOI: 10.1016/j.jhazmat.2019.122001

6. Zhu C, Liu D, Chen Z, Li L, You T. An Ultra-Sensitive Aptasensor Based on Carbon Nanohorns/Gold Nanoparticles Composites for Impedimetric Detection of Carbendazim at Picogram Levels. Journal of Colloid and Interface Science. 2019;(546):92-100. DOI: 10.1016/j. jcis.2019.03.035.

\section{Author information}

Anastasia V. Shabalina, senior researcher, Tomsk State University; Address: 1, Novosobornaya sq., Tomsk, Russian Federation 634050; Phone: +7(382)2531591; e-mail: shabalinaav@gmail.com, http://orcid.org/0000-0003-4741-2398

Received 26 February 2021 Revision Received 18 March 2021 Accepted 31 March 2021 